\title{
Exposure related mutagens in urine of rubber workers associated with inhalable particulate and dermal exposure
}

\author{
R Vermeulen, R P Bos, J Pertijs, H Kromhout
}

See end of article for authors' affiliations

.....................

Correspondence to: Dr H Kromhout, Environmental and Occupational Health Division, Institute of Risk Assessment Sciences, Utrecht University, PO Box 80176, 3508 TD Utrecht Netherlands;

H.Kromhouや@iras.uu.nl

Accepted 14 May 2002

\begin{abstract}
Aims: To determine the relation of the inhalation and dermal exposure routes and mutagenic activity in the urine of rubber workers $(n=105)$.

Methods: Mutagenic activity of ambient total suspended particulate matter (TSPM), surface contamination wipes, and Sunday and weekday urine samples was assessed with S typhimurium YG1041 in the presence of a metabolic activation system. Each subject was grouped into one of two exposure categories for dermal exposure (high $\left(\geqslant 25\right.$ revertants $\left./ \mathrm{cm}^{2}\right)$, low $\left(<25\right.$ revertants $\left.\left./ \mathrm{cm}^{2}\right)\right)$ based on the mutagenic activity detected on likely skin contact surfaces and into two airborne mutagenic exposure categories (high $\left(\geqslant 210\right.$ revertants $\left./ \mathrm{m}^{3}\right)$, low $\left(<210\right.$ revertants $\left.\left./ \mathrm{m}^{3}\right)\right)$. The potential influence of skin aberrations and acetylation status (NAT2) on urinary mutagenicity levels was also evaluated.

Results: A non-significant increase of +1605 revertants/g creatinine in urinary mutagenicity during the workweek relative to levels observed on Sunday was observed for the total population. Subsequent multivariate regression analyses, with the subjects' weekday urinary mutagenicity levels as the dependent variable, revealed associations with environmental and mainstream tobacco smoke exposure, with the level of mutagenic contamination on surfaces with which the subjects had likely contact, with the subjects' inhalable particulate exposure level, with observed mild skin aberrations, and when the subjects had a slow acetylation phenotype. Similar associations, although weaker were observed with Sunday urinary mutagenicity levels as well, except for the association with slow acetylation phenotype. Based on measured exposure levels it could be estimated that a high potential for exposure to surface contamination with mutagenic activity increased weekday urinary mutagenicity by about $62 \%$ when compared to low exposed workers, while high inhalable particulate exposure levels increased weekday urinary mutagenicity levels by about $21 \%$. Subjects with mild skin aberrations had an additional, non-significant, increase in weekday urinary mutagenic activity compared to subjects without any skin aberrations.

Discussion: Results suggest that the dermal exposure route may contribute more to the level of genotoxic compounds in urine of rubber workers than the inhalation route. Although the study was limited in size, the results warrant further investigation in the importance of and ways to effectively control the dermal exposure route in the rubber industry.
\end{abstract}

m n several studies in the rubber manufacturing industry, mutagenic activity has been detected in chemicals used as raw materials, in airborne rubber dust and fume samples taken from the workplace, and in the urine of exposed rubber workers. ${ }^{1}$ Although increased urinary mutagenicity levels have been detected in these workers, direct relations with external exposure levels have seldom been identified..$^{2-4}$ Only in a study of workers in an aircraft tyre retreading company was a direct relation between urinary mutagenicity and dermal exposure to cyclohexane soluble matter (CSM) found. ${ }^{4}$ The suggestion that dermal exposure could be an important exposure route in the rubber industry has also been supported by the observation that the amount of CSM potentially available for uptake through the skin could be up to 10 times higher than through inhalation. ${ }^{5}$

Recently, skin contact with contaminated surfaces and deposition of airborne contaminants on the skin were identified as important exposure determinants of dermal exposure to total organic matter in the rubber manufacturing industry. ${ }^{56}$ Moreover, substantial mutagenic activity was detected on these contact surfaces, making dermal exposure to mutagenic compounds in the rubber manufacturing industry more than likely. ${ }^{7}$ Although it is clear that substantial dermal exposure to mutagenic compounds occurs, no quantitative data are available on the actual dermal uptake of these compounds. Therefore, the relative contribution of the dermal exposure route in relation to the inhalation route for genotoxic exposures in the rubber manufacturing industry remains unclear.

Dermal absorption depends in general on the chemical and physical properties of the contaminant and on the actual concentration of the contaminant in the aqueous layer on the skin. Impairment of the skin barrier function can, however, drastically increase dermal uptake. ${ }^{8-10}$ Skin aberrations, such as hand dermatitis and traumata of the skin, are a common occurrence in the rubber manufacturing industry, with prevalence rates for hand dermatitis up to $35 \%$ among rubber workers. ${ }^{11}$ The high prevalence of skin impairments in this industry could potentially enhance dermal uptake and thereby increasing the relative importance of the dermal route in this industry.

This study was conducted to determine the relation between the inhalation and dermal exposure routes and mutagenic activity in urine of rubber workers, who were

Abbreviations: CSM, cyclohexane soluble material; DMSO, dimethylsulphoxide; ETS, environmental tobacco smoke; TSPM, total suspended particulate matter 
selected from a large cross-industry survey in the Netherlands. ${ }^{11}{ }^{12}$ Mutagenic activity of ambient total suspended particulate matter (TSPM), surface contamination, and Sunday and weekday urine samples was assessed with Salmonella typhimurium YG1041 in the presence of a metabolic activation system. The potential influence of skin aberrations and acetylation status (NAT2) on urinary mutagenicity levels was also evaluated.

\section{MATERIALS AND METHODS Study population}

Selection of the study population is described in detail elsewhere. ${ }^{12}{ }^{13}$ In short, 224 male subjects, employed in nine rubber manufacturing companies (three rubber tyre, five general rubber goods, and one retreading company), were randomly selected based on their production function (for example, compounding and mixing, pretreating, moulding, curing, finishing, shipping, engineering service, and laboratory). Sample and data collection was conducted from January to July 1997 and was carried out within one week for each company; it comprised a medical survey, area and personal exposure measurements, and collection of biological samples. Informed consent was obtained after the study was explained to the subjects.

Each subject was grouped into one of four a priori exposure groups defined by: (1) dermal exposure, based on the median levels of mutagenic activity detected on likely skin contact surfaces (high $\left(\geqslant 25 \mathrm{rev} / \mathrm{cm}^{2}\right)$, low $\left.\left(<25 \mathrm{rev} / \mathrm{cm}^{2}\right)\right)$; and (2) airborne exposure, based on the median levels of mutagenic activity detected in air samples (high $\left(\geqslant 210 \mathrm{rev} / \mathrm{m}^{3}\right)$, low $\left.\left(<210 \mathrm{rev} / \mathrm{m}^{3}\right)\right)$. The four exposure groups are characterised as follows:

(a) Low TSPM mutagenicity and low surface mutagenic exposure levels (LL)

(b) High TSPM mutagenicity and low surface mutagenic exposure levels (HL)

(c) Low TSPM mutagenicity and high surface mutagenic exposure levels $(\mathrm{LH})$

(d) High TSPM mutagenicity and high surface mutagenic exposure levels $(\mathrm{HH})$.

No subjects matched the a priori categorisation of low TSPM mutagenicity and high surface mutagenic exposure levels (LH), leaving only three exposure groups (LL, HL, HH). From each of these exposure groups 20 non-smokers were randomly selected. Additionally, 20 current smokers were selected from both the LL and the HH exposure group. For subjects employed as technical engineers $(n=16)$ the work practices were too diverse to enable identification of relevant TSPM and surface wipe samples. However, technical engineers are workers with a potential for high dermal exposure levels and therefore were included in the present survey as a separate category. All subjects were full time employed.

Subjects were administered a questionnaire, as part of the initial survey to collect information regarding age, medical history, personal hygiene, and lifestyle factors. Mainstream tobacco smoke and environmental tobacco smoke (ETS) intake was evaluated by assessing cotinine levels in both the Sunday and weekday urine samples. ${ }^{14}$ Cotinine in urine was quantified by high performance liquid chromatography according to the method of Barlow and colleagues, ${ }^{15}$ using the modifications described by Parviainen and Barlow. ${ }^{16}$ Creatinine levels were used to estimate urinary dilution using a colometric test, based on the Jaffé reaction between creatinine and sodium picrate. Cotinine levels were expressed as $\mu \mathrm{g} / \mathrm{g}$ creatinine.

\section{Ambient exposure measures}

Ambient exposure measurements were collected as described previously. ${ }^{617}$ Briefly, TSPM exposure was measured on random selected days (Tuesday to Thursday) using a high volume sampler. ${ }^{18}$ On average 1.8 samples were collected for each department within a company; samples within a department were subsequently pooled for mutagenicity analysis. Surface contamination was estimated by obtaining wipe samples of potential contact surfaces. Samples were taken by a modification of the OSHA wipe sampling procedure. ${ }^{6}{ }^{19}$ In the modified procedure, a surface area of $100 \mathrm{~cm}^{2}$ of a potential contact surface was chosen as sampling area. The area was wiped three times consecutively with Clean cylce wet VDU wipes (Inmac) containing 70\% water and 30\% isopropyl alcohol.

\section{Personal exposure measures}

Personal exposure to inhalable particulate was measured on three consecutive days (Tuesday, Wednesday, and Thursday) by means of a PAS6 sampling head mounted near the breathing zone of the worker. ${ }^{18}{ }^{20}$ Personal dermal exposure to CSM was measured with a dermal pad sampler worn at the lower part of the wrist of the hand of preference on the same days as the exposure to inhalable particulate was measured. ${ }^{521}$

CSM contents of inhalable and dermal exposure samples were determined by the NIOSH PCAM 217 method. ${ }^{20}{ }^{22}$ In short, samples were placed in cyclohexane (Merck, Darmstadt, Germany) and sonicated for 20 minutes. Suspension was filtered through a glassintertube G4 (Schott, Germany) and collected in a preweighed $10 \mathrm{ml}$ vial. After evaporation of cyclohexane under nitrogen and subsequent drying for two hours at $40^{\circ} \mathrm{C}$ the residue was weighed by means of a microbalance. ${ }^{12}$ No additional chemical analysis of the extract was performed.

\section{Mutagenicity assays}

TSPM and surface contamination samples were extracted consecutively with cyclohexane (Merck), dichloromethane (Merck) and methanol (Merck) as described previously. ${ }^{17}$ After evaporation of organic solvents under nitrogen at $40^{\circ} \mathrm{C}$, the residue was dissolved in $2.5 \mathrm{ml}$ of dimethylsulphoxide (DMSO) (Merck). ${ }^{4}$

Spot urine samples were collected on Sunday, Wednesday, and Thursday at approximately the same time of the day $( \pm 4$ $\mathrm{pm}$ ). Volumes, corresponding to $0.5 \mathrm{mmol}$ of creatinine of the Wednesday and Thursday post-shift urine sample were pooled, resulting in a volume corresponding to $1.0 \mathrm{mmol}$ of creatinine. Urine aliquots corresponding to $1.0 \mathrm{mmol}$ of creatinine from Sunday and the pooled weekday urine sample were neutralised to $\mathrm{pH} 7.0$ and extracted with XAD-2 resin (6 $\mathrm{cm}^{3}$ bed volume) and eluted with $10 \mathrm{ml}$ of acetone. After evaporation at $40^{\circ} \mathrm{C}$, the residue was dissolved in $2.5 \mathrm{ml}$ of DMSO. $^{4}$

TSPM $(n=83)$, surface contamination $(n=104)$, and urine $(\mathrm{n}=232)$ extracts were assayed for mutagenic activity with Salmonella typhimurium YG1041 with S9 mix of aroclor 1254 induced rat liver (mean mutagenicity of references: spontaneous 144 (SD 19) rev/plate; positive control (2aminopyrene, $0.1 \mu \mathrm{g} / \mathrm{plate}$ ), mean 2584 (SD 232) rev/ plate). ${ }^{23}{ }^{24}$ DMSO extracts were assayed at five different dose levels in triplicate. For determination of mutagenic activity, the mean number of revertants was calculated for each dose level and subsequently a dose-response curve was constructed. The slope of the linear component was used as an estimate of mutagenic potency. ${ }^{25}$ Mutagenicity of TSPM and surface contamination was expressed as revertants $/ \mathrm{m}^{3}$ (rev/ $\left.\mathrm{m}^{3}\right)$ and as revertants $/ \mathrm{cm}^{2}\left(\mathrm{rev} / \mathrm{cm}^{2}\right)$, respectively. Urinary mutagenic activity was expressed as revertants/g creatinine (rev/g creat).

\section{Acetylation status}

An aliquot of the Wednesday post-shift urine sample was taken and analysed for caffeine and metabolites, according to the procedure described by Grant and colleagues. ${ }^{26}$ Designation of the acetylator (NAT2) phenotype was based on the 
Table 1 Overview of selected characteristics of study subjects and summary statistics of exposure parameters

\begin{tabular}{ll}
\hline Characteristics of study subjects & \\
\hline $\mathrm{n}$ & 105 \\
Mean age, y (SD) & $37.9(9.0)$ \\
$\%$ Male & 100 \\
$\%$ Current smokers & 36.2 \\
$\%$ Major skin complaints & 5.7 \\
$\%$ Minor skin complaints & 34.3 \\
$\%$ Slow acetylation & 61.0
\end{tabular}

Summary statistics of exposure parameters GM (GSD)

Personal inhalable particulate $\left(\mathrm{mg} / \mathrm{m}^{3}\right)$

Personal dermal exposure $\left(\mu \mathrm{g} / \mathrm{cm}^{2}\right) \quad 49.7(2.51)$

Ambient particulate matter (TSPM) $\left(\mathrm{mg} / \mathrm{m}^{3}\right) \quad 0.15(2.10)$

TSPM mutagenicity $\left(\mathrm{rev} / \mathrm{m}^{3}\right)$

Surface mutagenicity $\left(\mathrm{rev} / \mathrm{cm}^{2}\right)$

$175.4(3.68)$

$40.2(3.11)$

GM, geometric mean; GSD, geometric standard deviation

ratio between 5-acetylamino-6-formylamino-3-methyluracil (AFMU) and 1-methylxanthine (IX). Results showed a clear bimodality of $\mathrm{N}$-acetylation capacity with a ratio cut off point of 1.7 resulting in $61 \%$ slow and $39 \%$ fast acetylators.

\section{Skin aberrations}

Two dermatologists conducted a medical evaluation of current skin conditions. ${ }^{13}$ Objective skin symptoms were used for the classification: active hand dermatitis ("major" dermatitis) $(\mathrm{n}=6)$; the first symptoms of dermatitis ("minor" dermatitis) $(\mathrm{n}=25)$; and skin injuries (traumata) $(\mathrm{n}=17) \cdot{ }^{27}{ }^{28}$ In the statistical analyses, subjects with minor dermatitis and/or traumata at the skin were classified as subjects with mild skin aberrations $(\mathrm{n}=36)$. Subjects with "major" hand dermatitis with or without traumata were regarded as a separate group in these analyses, as in a previous study it was observed that workers with a clear eczematous skin ("major" hand dermatitis) consciously avoid skin aggravating activities, including several work practices. ${ }^{11}$

\section{Calculations and statistics}

Mean personal inhalable particulate, inhalable CSM and dermal CSM exposure was estimated using the James-Stein shrinkage estimator to maximise accuracy and precision of the exposure estimates. ${ }^{29-31}$ In this computation a weighted mean $\left(\Theta_{\mathrm{gi}}\right)$ of the individual $\left(z_{\mathrm{gi}}\right)$ and group mean $\left(z_{\mathrm{g}}\right)$ exposure is calculated based on a weighting factor $\left(B_{\mathrm{g}}\right)$ that determines the optimal combination of the group and individual means (equation 1). Subjects were grouped by production function $(\mathrm{n}=8)$ and subsequently group means were calculated based on the individual mean exposures derived from the repeated personal exposure measurements. Within $\left(S_{w w}^{2}\right)$ and between worker $\left(S_{b w}^{2}\right)$ components of variance were estimated from the ln transformed exposure concentrations employing a one way nested random effects ANOVA model for each production function separately. The weighting factor $\left(\mathrm{B}_{\mathrm{g}}\right)$ was estimated for a fixed number of repeated measurements $(\mathrm{J}=3)$ on each individual using equation 2.

$\Theta_{g i}=\left(B_{g}\right) \bar{z}_{g}+\left(1-B_{g}\right) \bar{z}_{g i}$

$\hat{B}_{g}=\frac{\left(S_{w w}^{2}\right) J^{-1}}{S_{b w}^{2}+\left(S_{w w}^{2}\right) J^{-1}}$

Surface mutagenic contamination was calculated by averaging the mutagenic activity levels of contact surfaces relevant for each subject. In these calculations, surface wipe samples were weighed equally regardless of the frequency and duration of contact with the particular surface. Mean ambient TSPM mutagenicity was calculated based on the pooled TSPM samples according to the work areas the subject was employed in. Cotinine corrected Sunday and pooled weekday urinary mutagenicity levels were calculated based on the algorithms found for the relation between the natural logarithms of urinary cotinine and urinary mutagenicity levels described previously. $^{14}$

Differences in geometric means of cotinine corrected mutagenicity levels between Sunday and weekdays for the total population and the a priori defined exposure groups were tested with Student's $t$ test. Finally, a multivariate regression model was built to identify factors which contributed significantly to unadjusted ln transformed urinary mutagenicity levels. The results are presented separately for the Sunday and weekday urine samples. Possible differences in the regression coefficients $(\beta)$ of the identified determinants between the Sunday and weekday urinary mutagenicity levels were subsequently studied in a mixed effect model with the inclusion of an interaction term between day (Sunday or weekday) and the identified determinants. The random effects part of the mixed effect model was used to allow for the fact that Sunday and weekday urinary mutagenicity levels were derived from repeated urine samples of the same subject. All statistical analyses were performed using SAS version 6.12 software. $^{32}$

\section{RESULTS}

Of the 116 selected subjects, 105 (90.5\%) had complete data concerning exposure estimates, current skin condition, urinary cotinine, and urinary mutagenicity levels. No systematic differences (as far as available) in smoking habits, urinary cotinine, and mutagenic activity levels were observed for subjects with incomplete data $(n=11)$ or those with complete data $(n=105)$. Subsequently, results presented in this paper are based on these 105 subjects. Table 1 presents an overview of selected characteristics of the study subjects and summary statistics of the exposure parameters.

Table 2 Mean mutagenic activity of total suspended particulate matter (TSPM) $\left(\mathrm{rev} / \mathrm{m}^{3}\right)$ and mutagenicity of surface contamination $\left(\mathrm{rev} / \mathrm{cm}^{2}\right)$ levels for the four a priori defined exposure groups

\begin{tabular}{lllc}
\hline & & \multicolumn{2}{l}{ Mutagenicity } \\
\cline { 3 - 4 } Exposure group & NS/S* & $\begin{array}{l}\text { TSPM GM } \\
\text { (GSD) }\end{array}$ & $\begin{array}{c}\text { Surface GM } \\
\text { (GSD) }\end{array}$ \\
\hline Low TSPM and low surface mutagenic exposure levels (LL) & $18 / 18$ & $73(2.13)$ & $19(1.02)$ \\
High TSPM and low surface mutagenic exposure levels (HL) & $19 / 0$ & $449(1.73)$ & $20(1.12)$ \\
High TSPM and high surface mutagenic exposure levels (HH) & $20 / 16$ & $514(1.73)$ & $147(2.60)$ \\
Technical engineers (TD) & $10 / 4$ & NQ & NQ \\
\hline
\end{tabular}

*Number of subjects. NS, non-smokers; $S$, current smokers.

†Geometric mean (GM) and geometric standard deviation (GSD) of mutagenic exposure levels. $N Q$, not quantified. 

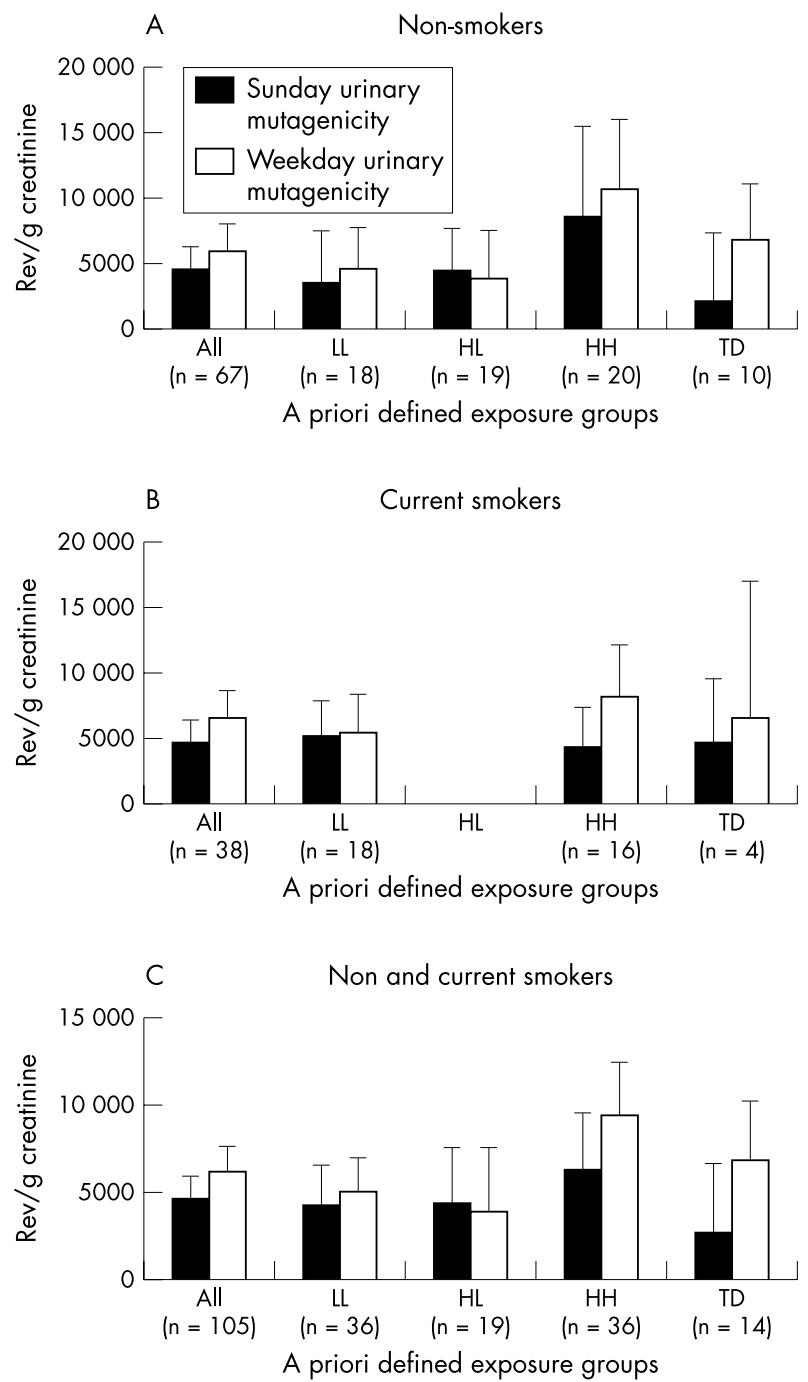

Figure 1 Geometric mean and $95 \%$ upper confidence limit of cotinine corrected urinary mutagenicity levels (rev/g creatinine) for the four a priori defined exposure groups.

Table 2 presents mean mutagenic TSPM and surface contamination levels for the four a priori defined exposure groups. The exposure groups HL and $\mathrm{HH}$ both had significantly higher TSPM mutagenicity levels than exposure group
LL ( $t$ test, $\mathrm{p}<0.0001$ ). Exposure group $\mathrm{HH}$ exhibited significantly higher surface mutagenic contamination levels than exposure groups LL and HL ( $t$ test, $\mathrm{p}<0.0001$ ). No statistical difference was observed between the exposure groups $\mathrm{HL}$ and $\mathrm{HH}$ and between exposure groups LL and HL with regard to respectively TSPM and surface mutagenicity. These results confirm the intended differences (contrast) in ambient mutagenic exposure profiles between the four a priori defined exposure groups.

Figure l shows geometric means of cotinine corrected urinary mutagenicity levels for the a priori defined exposure groups. Non-smokers (A) and current-smokers (B) had similar patterns in Sunday and weekday urinary mutagenicity for the different exposure groups, with the exception of a borderline significant difference in cotinine corrected Sunday urinary mutagenicity levels between smokers and nonsmokers within the exposure group $\mathrm{HH}(t$ test, $\mathrm{p}=0.10$ ). To increase the number of subjects per exposure group, smokers and non-smokers were subsequently pooled (C). A nonsignificant increase of $+1605 \mathrm{rev} / \mathrm{g}$ creat in urinary mutagenicity during the workweek relative to levels observed on Sunday was observed for the total population ( $t$ test, $\mathrm{p}=0.08)$. The increase was most pronounced among technical engineers $(+4196 \mathrm{rev} / \mathrm{g}$ creat; $t$ test, $\mathrm{p}=0.08)$ and subjects who were exposed to high levels of airborne mutagenicity levels and had a high potential for dermal contact with surfaces contaminated with substances exhibiting mutagenic activity $(+3206 \mathrm{rev} / \mathrm{g}$ creat; $t$ test, $\mathrm{p}=0.12)$.

Multivariate regression analyses revealed that the exposure group $\mathrm{HH}$ had an almost twofold increase $(\beta=0.64$ and $\left.\mathrm{e}^{0.64}=1.9 ; \mathrm{p}=0.01\right)$ in weekday urinary mutagenicity levels relative to the LL exposure group (table 3) while no statistically significant differences in Sunday urinary mutagenicity levels between these exposure groups could be detected. In addition, slow acetylation phenotype $(\beta=0.44$; $\mathrm{p}=0.04)$ and mild skin aberrations $(\beta=0.42 ; \mathrm{p}=0.06)$ was also found to be associated with increased weekday urinary mutagenicity levels. Interestingly, subjects with mild skin aberrations tended to also have higher Sunday urinary mutagenicity levels $(\beta=0.50 ; p=0.09)$. Further statistical analyses with area and personal exposure measures as continuous variables resulted in the model presented in table 4 . In concordance with earlier results a strong association was observed between surface mutagenicity and urinary mutagens while TSPM mutagenicity exhibited no apparent association. Personal inhalable particulate exposure was also found to be associated with increased urinary mutagenicity levels. Interestingly, the observed associations between the exposure measures and weekday urinary mutagenicity were statistically not different from the observed relations between these exposure measures and Sunday urinary mutagenicity levels, except

Table 3 Relation between urinary cotinine, NAT2 phenotype, skin aberrations, and the a priori defined exposure groups and Sunday and weekday urinary mutagenicity levels (rev/g creatinine) of rubber manufacturing workers $(n=105)$

\begin{tabular}{|c|c|c|c|c|}
\hline & \multicolumn{2}{|c|}{ Sunday urinary mutagenicity } & \multicolumn{2}{|c|}{ Weekday urinary mutagenicity } \\
\hline & $\beta$ (SE)* & $\mathrm{p}$ value & $\beta(\mathrm{SE})^{*}$ & $\mathrm{p}$ value \\
\hline Intercept & $8.25(0.35)$ & $<0.0001$ & $8.59(0.26)$ & $<0.0001$ \\
\hline Urinary cotinine ( $\mu \mathrm{g} / \mathrm{g}$ creatinine) & $0.27(0.05)$ & $<0.0001$ & $0.26(0.04)$ & $<0.0001$ \\
\hline Slow acetylation (NAT2) $†$ & $0.06(0.28)$ & 0.83 & $0.44(0.21)$ & 0.04 \\
\hline Mild skin aberrations $\ddagger \S$ & $0.50(0.30)$ & 0.09 & $0.42(0.22)$ & 0.06 \\
\hline Major dermatitis§ & $0.04(0.61)$ & 0.95 & $0.05(0.45)$ & 0.91 \\
\hline High TSPM mutagenicity and low surface mutagenicity $(\mathrm{HL}) \uparrow$ & $0.05(0.43)$ & 0.91 & $-0.20(0.31)$ & 0.52 \\
\hline High TSPM mutagenicity and high surface mutagenicity $(\mathrm{HH}) \boldsymbol{\uparrow}$ & $0.41(0.33)$ & 0.22 & $0.64(0.25)$ & 0.01 \\
\hline Technical service (TD) & $-0.51(0.44)$ & 0.26 & $0.29(0.33)$ & 0.39 \\
\hline
\end{tabular}

* $\beta$ coefficient and standard error of estimate.

†Relative to fast acetylation phenotype.

$\ddagger$ Minor dermatitis and/or traumata of the skin.

$\S$ Relative to subjects with no skin aberrations at time of the study.

IRelative to low TSPM mutagenicity and low surface mutagenicity (LL) exposure group. 
Table 4 Relation between urinary cotinine, NAT2 phenotype, mild skin aberrations, and In transformed exposure measures and Sunday and weekday urinary mutagenicity (rev/g creatinine) of rubber manufacturing workers, excluding technical engineers* $(n=91)$

\begin{tabular}{|c|c|c|c|c|}
\hline & \multicolumn{2}{|c|}{ Sunday urinary mutagenicity } & \multicolumn{2}{|c|}{ Weekday urinary mutagenicity } \\
\hline & $\beta(\mathrm{SE}) \dagger$ & $\mathrm{p}$ value & $\beta(\mathrm{SE}) \dagger$ & $\mathrm{p}$ value \\
\hline Intercept & $8.02(0.51)$ & $<0.0001$ & $8.09(0.42)$ & $<0.0001$ \\
\hline Urinary cotinine ( $\mu \mathrm{g} / \mathrm{g}$ creatinine) & $0.22(0.05)$ & $<0.0001$ & $0.24(0.04)$ & $<0.0001$ \\
\hline Slow acetylation (NAT2) $\ddagger$ & $0.11(0.29)$ & 0.70 & $0.54(0.23)$ & 0.02 \\
\hline Mild skin aberrations§ & $0.42(0.30)$ & 0.15 & $0.37(0.24)$ & 0.12 \\
\hline Inhalable particulate $\left(\mathrm{mg} / \mathrm{m}^{3}\right)$ & $0.40(0.22)$ & 0.07 & $0.42(0.18)$ & 0.02 \\
\hline Surface mutagenicity $\left(\mathrm{rev} / \mathrm{cm}^{2}\right)$ & $0.22(0.12)$ & 0.07 & $0.28(0.10)$ & 0.005 \\
\hline TSPM mutagenicity $\left(\mathrm{rev} / \mathrm{m}^{3}\right) \boldsymbol{\Phi}$ & $0.07(0.13)$ & 0.61 & $0.11(0.14)$ & 0.44 \\
\hline
\end{tabular}

*Technical engineers were not included in the analysis due to missing ambient mutagenic exposure estimates. $\dagger \beta$ coefficient and standard error of estimate. $\ddagger$ Relative to fast acetylation phenotype.

$\S M$ Minor dermatitis and/or traumata of the skin.

IEffect estimates presented for TSPM mutagenicity are from the univariate analysis; TSPM mutagenicity was not included in the multivariate analysis to limit the degrees of freedom used in the analysis and because of the moderate correlation between TSPM and surface mutagenicity ( $r=0.49)$.

for slow acetylation phenotype which only exhibited a significant relation with the weekday urinary mutagenicity levels.

\section{DISCUSSION AND CONCLUSIONS}

We studied mutagens in urine of rubber workers in relation to inhalable and dermal exposure. In addition, the potential influence of skin aberrations and acetylation status on urinary mutagenicity levels was addressed. Inhalable and dermal exposure assessment was based on area and personal exposure measurements. Area measurements were used to assess potential mutagenic exposure conditions encountered in the rubber manufacturing industry as personal exposure samples did not result in enough collected material to be tested in the Ames mutagenicity assay. The collected TSPM and surface contamination samples were tested for mutagenicity with $S$ typhimurium YG104l in the presence of a metabolic activation system. The strain YG1041, with elevated levels of nitroreductase and $O$-acetyltransferase, was shown in a previous study to be highly sensitive for detecting mutagenicity in rubber dust and fume samples. ${ }^{17}$

Assignment of ambient mutagenic exposure levels to the individuals was based on the work area the subject was employed in and actual tasks performed. Mean individual TSPM and surface mutagenicity levels were calculated independent of duration of contact with a particular surface or residence time in a particular work area. Therefore these estimates could have been imprecise, as indicated by the moderate but significant correlation between personal inhalable and ambient particulate matter concentrations $(r=0.55$, $\mathrm{p}<0.0001$ ). However, TSPM and surface mutagenic exposure levels are to a large extent determined by company specific factors and to a lesser extent by production functions within a company. ${ }^{7}$ Although individuals may work in different areas they do not work in more than one production function or company. Therefore, exposure misclassification in individual estimates of external mutagenic exposure conditions based on ambient mutagenic TSPM and surface contamination levels will most likely have been limited, resulting in a justifiable relative ranking between subjects from different companies and production functions.

Personal exposure estimates of inhalable particulate, inhalable CSM, and dermal CSM exposure were calculated based on a weighted mean of the individual and group mean exposure to maximise accuracy and precision of the exposure estimates. ${ }^{31}$ Estimates of exposure based on individual means may result in attenuation of the exposure-response relation while grouped estimates may control attenuation bias but may result in a decrease of the precision. ${ }^{33}{ }^{34}$ Combining individual and group estimates can simultaneously control both types of error. The variability in personal exposure measures was almost equally distributed between and within subjects. The James-Stein exposure estimate was therefore largely based on the calculated mean individual exposure estimates (median weighing factor $\left(\mathrm{B}_{\mathrm{g}}\right)$ for inhalable particulate, inhalable CSM, and dermal CSM was respectively $0.19,0.22$, and 0.27 ). Not surprisingly, additional statistical analyses using the calculated mean individual exposure levels, for example without shrinkage to the group mean, showed a similar, but weaker association between inhalable particulate and urinary mutagenicity levels (not shown).

The results of this study showed a borderline statistical significant increase in urinary mutagens of rubber workers during the workweek relative to Sunday. The increase in urinary mutagenicity was most pronounced among technical engineers and subjects with potentially high TSPM and surface mutagenic exposure levels. No increase in urinary mutagenicity was observed among subjects with a high TSPM but low surface mutagenic activity. Further statistical analyses using mutagenic activity of contact surfaces as a continuous variable confirmed the relation between mutagenicity of likely contact surfaces and urinary mutagenicity levels, while again no association was found between TSPM mutagenic activity levels and mutagens in urine of rubber workers. However, personal particulate exposure estimates were found to be associated with increased urinary mutagenicity levels. A similar association was also observed with ambient particulate exposure estimates and urinary mutagenicity (not shown). The fact that an association was found with the mass of the airborne particulate exposure but not with the mutagenic activity of the airborne particulates suggests the existence of non-extractable particle bound mutagenicity. This hypothesis is strengthened by the fact that no association was found between the mass of the extractable organic matter (inhalable CSM exposure) and urinary mutagenicity (not shown). The existence of non-extractable bound bioactive material to the surface of airborne rubber dust particles was also shown previously in a study by Alink and colleagues. ${ }^{35}$ In their study, $83 \%$ of the inhibition of the gap-junctional intercellular communication could be attributed to bioactive material tightly bound to the surface of the particulate matter, while only $17 \%$ of the observed effect could be attributed to bioactive material present in the extract. As a result, the a priori defined exposure groups might have had limited value with regard to airborne mutagenic exposure conditions.

Identified associations between occupational exposure estimates and urinary mutagenicity were not statistically 
different for the pooled weekday urine samples and Sunday urine samples, though in most instances the observed associations with Sunday urinary mutagenicity were statistically weaker. Urinary mutagens from workplace exposure in the Sunday urine collection could originate from inhaled particles trapped in the lung or in pores of the skin resulting in prolonged exposure and/or due to a long half life of the detected mutagens. As significant occupational related (residual) urinary mutagenic activity was detected in the Sunday urine samples; these samples cannot be regarded as "clean" baseline samples and therefore cannot be used as a subject's own control. Consequently, the use of the ratio or absolute difference between Sunday and weekday urinary mutagenicity levels would have resulted in an underestimation of the mutagenic effect.

Subjects with mild skin aberrations ("minor" dermatitis and traumata) showed higher urinary mutagenicity levels than subjects with a normal skin barrier function. This association was stronger in the regression analyses that included the technical engineers (table 3 ). Technical engineers had a high prevalence of minor dermatitis and traumata of the skin, $33 \%$ and $22 \%$, respectively. Exclusion of this exposure group from subsequent regression analyses because of missing ambient mutagenic exposure estimates, resulted in the loss of statistical power and consequently the loss of statistical significance. In several volunteer studies it has been shown that mild skin barrier damage could dramatically increase percutaneous penetration..$^{86-38}$ However, the magnitude of the effect depends greatly on the chemical properties and clinical manifestation of the disease. ${ }^{39}$ In this study, an indication was found for an increase in uptake of genotoxic compounds among subjects with mild dermatitis and traumata of the skin at time of the survey, but not for subjects with major dermatitis. In a previous study it was shown that rubber workers often did not regard their impaired skin (for example, minor dermatitis) as a disease and therefore did not alter their working practice. ${ }^{13}$ However, in the case of major dermatitis changes in behaviour were observed with regard to skin aggravating risk factors. ${ }^{11}$ The results of this study corroborate that observation.

Slow acetylators had significantly higher weekday urinary mutagenicity levels than fast acetylators. NAT2 modulation of urinary mutagenicity has been found in several studies of subjects exposed to aromatic amines. ${ }^{40-42}$ In addition, slow acetylation status has been associated with an increase in urinary bladder cancer risk among subjects with past occupational exposure to aromatic amines. ${ }^{43}$ Remarkably, NAT2 phenotype only influenced urinary mutagenicity levels during the working week, while the other determinants significantly affected both weekday and Sunday urinary mutagenicity levels. The observed relation between urinary mutagenicity and the measured exposure (inhalable particulate, surface mutagenicity) were therefore probably not modulated by NAT2 dependent enzyme activities, which points towards other NAT2 modulated (occupational) exposures with a relative short half life that were not quantified as such with the used sampling and/or analytical techniques. This is corroborated by the fact that no interaction was observed between the exposure estimates and acetylation status on urinary mutagenicity.

Based on measured exposure levels (3rd quartile (Q3, high) versus 1st quartile (Q1, low)) and estimated effect $(\beta)$ it can be estimated that a high potential for exposure to surface contamination with mutagenic activity increased weekday urinary mutagenicity by about $62 \%$, while high inhalable particulate exposure levels increased weekday urinary mutagenicity by about $21 \%$.

The results of this study suggest that the dermal exposure route may contribute more to the level of genotoxic compounds in urine of rubber workers, than the inhalation route. The fact that subjects with mild skin aberrations possi-

\section{Main messages}

- Workers in the contemporary rubber industry are exposed to genotoxic compounds.

- The dermal exposure route may contribute more to the level of genotoxic compounds in urine of rubber workers than the inhalation route.

\section{Policy implications}

- Knowledge that dermal exposure contributes significantly to the level of mutagens in urine of rubber workers has important public health implications.

- Occupational epidemiological studies among workers employed in the rubber industry have focused on inhalation exposures and therefore possibly overlooked a significant exposure pathway

bly had an additional increase in urinary mutagenicity is important, as the prevalence rate of these unfavourable skin conditions is high in the population of workers in the rubber manufacturing industry. Although the study was limited in size, the results warrant further investigation into the importance of and ways to effectively control the dermal exposure route in the rubber manufacturing industry.

\section{ACKNOWLEDGEMENTS}

The authors are indebted to the employers and employees in the rubber manufacturing industry for close cooperation in this study. We would also like to acknowledge $\mathrm{J}$ de Hartog for assisting in data collection and H Wegh, T de Groot, and A Mom for the numerous chemical analyses. Prof. Dr Derk P Bruynzeel and Dr Edith M de Boer enabled the dermatological survey within the rubber study. Styphimurium strain YG104l was kindly obtained from Dr T Nohmi and Dr M Watanabe of the Division of Mutagenesis, National Institute of Genetics and Mutagenesis (Tokyo, Japan).

\section{Authors' affiliations}

R Vermeulen, H Kromhout, Environmental and Occupational Health Division, Institute of Risk Assessment Sciences, Utrecht University, Utrecht, Netherlands

R P Bos, J Pertijs, Department of Pharmacology \& Toxicology, UMC St Radboud, University of Nijmegen, Nijmegen, Netherlands

\section{REFERENCES}

1 Baranski B, Indulski J, Janik SE, et al. Mutagenicity of airborne particulates in the rubber industry. J Appl Toxicol 1989;9:389-93.

2 Crebelli R, Falcone E, Aquilina G, et al. Mutagenicity studies in a tyre plant: in-vitro activity of urine concentrates and rubber chemicals. IARC Sci Publ 1984;289-95.

3 Falck K, Sorsa M, Vainio $\mathrm{H}$, et al. Mutagenicity in urine of workers in rubber industry. Mutat Res 1980;79:45-52.

4 Bos RP, Kromhout H, lkink H, et al. Mutagens in urine of non-smoking and smoking workers in an aircraft tyre retreading plant. Skin exposure as a causal factor? Mutat Res 1989-223:41-8.

5 Kromhout H, Swuste P, Boleij JS. Empirical modelling of chemical exposure in the rubber-manufacturing industry. Ann Occup Hyg 1994;38:3-22

6 Vermeulen R, Heideman J, Bos RP, et al. Identification of dermal exposure pathways in the rubber manufacturing industry. Ann Occup Hyg 2000;44:551-60.

7 Vermeulen R, Bos RP, Kromhout H. Mutagenic exposure in the rubber manufacturing industry; an industry wide survey. Mutat Res 2001;490:27-34

8 Benfeldt E, Serup J, Menne T. Effect of barrier perturbation on cutaneous salicylic acid penetration in human skin: in vivo pharmacokinetics using microdialysis and non-invasive quantification of barrier function. $\mathrm{Br} J$ Dermatol 1999;140:739-48.

9 Hart TB. Paraquat-a review of safety in agricultural and horticultural use. Hum Toxicol 1987;6:13-18.

10 Yu XZ. [Biological monitoring of workers exposed to nitrofen and experimental study on its skin permeability]. Chung Hua Yu Fang I Hsueh Tsa Chih 1993;27:208-11.

11 Vermeulen R, Kromhout H, Bruynzeel DP, et al. Dermal exposure, hand washing and hand dermatitis in the rubber manufacturing industry. Epidemiology 2001;11:350-4. 
12 Vermeulen $\mathbf{R}$, de Hartog J, Swuste $P$, et al. Trends in exposure to inhalable particulate and dermal contamination in the rubber manufacturing industry: effectiveness of control measures implemented over a nine-year period. Ann Occup Hyg 2000;44:343-54.

13 Vermeulen R, Kromhout $H$, Bruynzeel DP, et al. Ascertainment of hand dermatitis using a symptom-based questionnaire; applicability in an industrial population. Contact Dermatitis 2000;42:202-6.

14 Vermeulen R, Wegh H, Bos RP, et al. Weekly patterns in smoking habits and influence on urinary cotinine and mutagenicity levels; confounding effect of non-smoking policies at the workplace. Cancer Epidemiol Biomarkers Prev 2000;9:1205-9.

15 Barlow RD, Thompson PA, Stone RB. Simultaneous determination of nicotine, cotinine and five additional nicotine metabolites in the urine of smokers using pre-column derivatisation and high-performance liquid chromatography. J Chromatogr 1987:419:375-80.

16 Parviainen MT, Barlow RD. Assessment of exposure to environmental tobacco smoke using a high-performance liquid chromatographic method for the simultaneous determination of nicotine and two of its metabolites in urine. J Chromatogr 1988;431:216-21.

17 Vermeulen R, Bos RP, de Hartog J, et al. Mutagenic profile of rubber dust and fume exposure in two rubber tire companies. Mutat Res 2000;468: 165-71

18 Kuile ter WM. Comparison of total dust measurements equipment at the workplace: Part II. Staub Reinhaltung der luft 1984;44:211-16.

19 Robbins MC. OSHA's wipe sampling policies and procedures. Sampling and analysis of toxic substances in the atmosphere-a symposium by ATSM. Boulder, CO: ATSM, 1979:79.

20 Kenny LC, Aitken R, Chalmers C, et al. A collaborative European study of personal inhalable aerosol sampler performance. Ann Occup Hyg 1997:41:135-53.

21 Durham WF, Wolff T. Measurement of the exposure of workers to pesticides. Bull WHO 1962;26:75-91.

22 NIOSH. Manual of analytical methods, 2 nd edn. Cincinnati, $\mathrm{OH}$ : US Department of Health, Education and Welfare, 1977:77-157-A.

23 Hagiwara $Y$, Watanabe $M$, Oda $Y$, et al. Specificity and sensitivity of Salmonella typhimurium YG1041 and YG1042 strains possessing elevated levels of both nitroreductase and acetyltransferase activity. Mutat Res 1993;291:171-80.

24 Maron DM, Ames BN. Revised methods for the Salmonella mutagenicity test. Mutat Res 1983;113:173-215.

25 Krewski D, Leroux BG, Creason J, et al. Sources of variation in the mutagenic potency of complex chemical mixtures based on the Salmonella/microsome assay. Mutat Res 1992;276:33-59.

26 Grant DM, Tang BK, Kalow W. A simple test for acetylator phenotype using caffeine. Br J Clin Pharmacol 1984;17:459-64.

27 Bruynzeel DP, Boer de EM, Brouwer EJ, et al. Dermatitis in bulb growers. Contact Dermatitis 1993;29:11-15.
28 Mei van der IA, Boer de EM, Bruynzeel DP. Contact dermatitis in Alstroemeria workers. Occup Med Oxf 1998;48:397-404.

29 James W, Stein C. Estimation with quadratic loss. Proceedings of the fourth Berkeley symposium on mathematical statistics and probability. Berkeley, CA: University of California Press, 1961:361-80.

30 Efron B, Morris C. Limiting the risk of Bayes and empirical Bays estimators. Part II: the empirical Bayes case. J Am Stat Assoc 1972;67:130-9

31 Seixas NS, Sheppard L. Maximizing accuracy and precision using individual and grouped exposure assessments. Scand J Work Environ Health 1996:22:94-101.

32 SAS Institute. SAS/STAT user's guide, version 6, 4th edn. Cary, NC: SAS Institute, Inc., 1990

33 Thomas D, Stram D, Dwyer J. Exposure measurement error: influence on exposure-disease. Relationships and methods of correction. Annu Rev Public Health 1993;14:69-93.

34 Tielemans E, Kupper LL, Kromhout H, et al. Individual-based and group-based occupational exposure assessment: some equations to evaluate different strategies. Ann Occup Hyg 1998;42:115-19.

35 Alink GM, Sjogren M, Bos RP, et al. Effect of airborne particles from selected indoor and outdoor environments on gap-junctional intercellula communication. Toxicol Lett 1998;96-97:209-13.

36 Aungst BJ, Blake JA, Hussain MA. Contributions of drug solubilization, partitioning, barrier disruption, and solvent permeation to the enhancement of skin permeation of various compounds with fatty acids and amines. Pharm Res 1990;7:712-18.

37 Gunther C, Kecskes A, Staks T, et al. Percutaneous absorption of methylprednisolone aceponate following topical application of Advantan lotion on intact, inflamed and stripped skin of male volunteers. Skin Pharmacol Appl Skin Physiol 1998;11:35-42.

38 Higo N, Hinz RS, Lau DT, et al. Cutaneous metabolism of nitroglycerin in vitro. II. Effects of skin condition and penetration enhancement. Pharm Res 1992;9:303-6.

39 Boman A, Wahlberg JE. Percutaneous absorption of 3 organic solvents in the guinea pig (I). Effect of physical and chemical injuries to the skin. Contact Dermatitis 1989:21:36-45.

40 Sinues B, Perez J, Bernal ML, et al. Urinary mutagenicity and $\mathrm{N}$-acetylation phenotype in textile industry workers exposed to arylamines. Cancer Res 1992;52:4885-9.

41 Bartsch H, Caporaso N, Coda M, et al. Carcinogen hemoglobin adducts, urinary mutagenicity, and metabolic phenotype in active and passive cigarette smokers. J Natl Cancer Inst 1990;82:1826-31.

42 Gabbani G, Hou SM, Nardini B, et al. GSTM1 and NAT2 genotypes and urinary mutagens in coke oven workers. Carcinogenesis 1996;17:1677-81

43 Marcus PM, Vineis $P$, Rothman N NAT2 slow acetylation and bladder cancer risk: a meta- analysis of 22 case-control studies conducted in the general population. Pharmacogenetics 2000;10:115-22. 